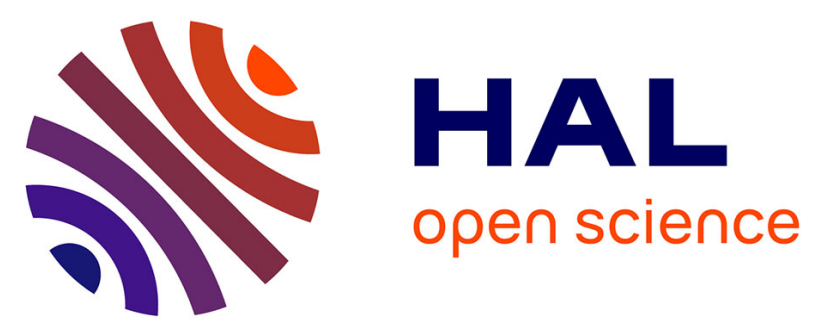

\title{
Correction par la fertilisation minérale des effets de l'ennoyage sur le blé d'hiver. II. - Expérimentation en culture hydroponique
}

Christian Guyot, Jean-Louis Prioul

\section{- To cite this version:}

Christian Guyot, Jean-Louis Prioul. Correction par la fertilisation minérale des effets de l'ennoyage sur le blé d'hiver. II. - Expérimentation en culture hydroponique. Agronomie, 1985, 5 (8), pp.751-759. hal-00884808

\section{HAL Id: hal-00884808 https://hal.science/hal-00884808}

Submitted on 1 Jan 1985

HAL is a multi-disciplinary open access archive for the deposit and dissemination of scientific research documents, whether they are published or not. The documents may come from teaching and research institutions in France or abroad, or from public or private research centers.
L'archive ouverte pluridisciplinaire HAL, est destinée au dépôt et à la diffusion de documents scientifiques de niveau recherche, publiés ou non, émanant des établissements d'enseignement et de recherche français ou étrangers, des laboratoires publics ou privés. 


\title{
Correction par la fertilisation minérale des effets de l'ennoyage sur le blé d'hiver. II. - Expérimentation en culture hydroponique
}

\author{
Christian GUYOT \& Jean-Louis PRIOUL $\left(^{*}\right)$ \\ Rhône-Poulenc Recherches, Centre de Recherches de la Croix de Berny, 182, avenue A.-Briand, \\ F 92160 Antony \\ $\left(^{*}\right)$ Adresse actuelle : Laboratoire Structure et Métabolisme des Plantes, associé au C.N.R.S. (L.A. 40), \\ Bâtiment 490, Université Paris-Sud, F 91405 Orsay Cedex
}

De jeunes plants de blé d'hiver, cultivés sur milieu liquide avec une privation d'oxygène, reproduisent tous les symptômes de l'ennoyage sur sol : arrêt de la croissance des racines séminales, développement rapide de racines adventives, diminution considérable de l'absorption ionique, carence minérale de la plante, diminution de la croissance foliaire.

La réduction du taux de croissance est due à une diminution des capacités de fixation photosynthétique. Lexritère le plus constant et caractéristique de l'asphyxie est la proportion de racines adventives dans la masse totale des racines ; la reprise de croissance après asphyxie concerne essentiellement le système racinaire, ce qui se fait partiellement au détriment des parties aériennes et en particulier du nombre de talles. La teneur en azote nitrique de la plante constitue également un marqueur intéressant car ses variations sont amplifiées par rapport à celle en azote total et révèlent un comportement différent des racines séminales et adventives.

Les effets de l'asphyxie racinaire sont très dépendants de la concentration en éléments nutritifs du milieu. Ils peuvent être effacés presque complètement avec des milieux optimaux, confirmant ainsi l'effet correcteur d'un apport d'engrais complet observé sur sol ; l'efficacité de cette pratique s'explique partiellement par le renforcement des flux ioniques passifs qui limitent la carence et atténuent la restriction de croissance.

Mots clés additionnels : Asphyxie racinaire, photosynthèse, croissance, morphogenèse.

Alleviation of the consequences of waterlogging in winter wheat seedlings by mineral fertilization. II. - Experiments in anaerobic solution culture.

Winter wheat seedlings grown in solution culture with a very low oxygen partial pressure showed all the symptoms of waterlogged plants in soil : inhibition of seedling root growth, fast development of adventitious roots, severe decrease in ion absorption, lower mineral content, restricted shoot growth. The reduction in growth rate was due to a decrease in photosynthetic fixation capacity. The more constant and characteristic criterion of root anaerobiosis was the proportion of adventitious roots. Growth recovery after the stress was mainly due to the root system, shoot growth and tiller number being lower than the control two weeks after reaeration. Nitric nitrogen content was a good index of plant reaction because its variations were greater than those of total nitrogen. For example, they revealed the different behaviour of seedling and adventitious roots. Sensitivity to root anaerobiosis was strongly dependent on the nutrient concentration of the root medium. Growth restriction was almost suppressed with optimized nutrient solutions, which confirms the corrective properties of fertilizer applications observed in soil culture. This practice can be explained by the increase in passive ionic fluxes, which limits the depletion in mineral content and alleviates the growth restriction.

Additional key words : Root anaerobiosis, photosynthesis, growth, morphogenesis. 


\section{INTRODUCTION}

La première partie de l'étude (Expérimentation sur sol, GUYOT \& PRIOUL, 1985) a permis de circonscrire les effets de l'ennoyage sur le blé d'hiver, de décrire la réponse post-asphyxique et de proposer des moyens de correction, la fertilisation renforcée. Néanmoins, la variabilité des réponses obtenues sur colonne de terre, liée probablement au défaut de maîtrise des conditions dans le sol, limite sérieusement les possibilités d'une analyse poussée qui permettrait de comprendre les mécanismes sous-jacents. Ceci nous a amenés à reprendre l'expérimentation en culture hydroponique.

Dans des conditions « asphyxiques » définies par MORISSET et al. (1982), les organes végétaux manifestent selon les espèces une grande diversité dans leur sensibilité et leur stratégie d'adaptation au défaut d'approvisionnement en oxygène des racines (ARMSTRONG, 1979). La plupart des espèces sensibles à l'ennoyage subissent, en conditions asphyxiantes, des réductions de croissance et de rendement associées à des symptômes caractéristiques : perte de turgescence, carence minérale et sénescence des parties aériennes; inhibition de la croissance, de l'absorption ionique et apparition de lésions racinaires. Ces symptômes ont d'abord été attribués aux changements qui se produisent dans un sol asphyxiant : accumulation de $\mathrm{CO}_{2}$, d'éthylène et de produits organiques toxiques (DREW, 1983). Cependant, la plupart des effets de l'ennoyage ont été reproduits en culture hydroponique par simple restriction de l'approvisionnement en oxygène des racines (TROUGHT \& DREW, 1980).

L'émission de racines adventives dans la région proche de la surface du sol est considérée comme un facteur essentiel de survie. Ces racines, plus «poreuses» que les racines primaires, possèdent une structure corticale lacunaire (aérenchymes) paraissant autoriser leur approvisionnement en oxygène gazeux par " transport interne » à partir des parties aériennes; ces racines sont capables de croître en solution non aérée ou en sol inondé (LUXMOORE \& STOLZY, 1969).

L'objet de la présente étude a été de vérifier au moyen de cultures hydroponiques que les effets de l'ennoyage s'identifient pour l'essentiel à une privation d'oxygène au niveau racinaire. A l'aide de ce modèle expérimental bien défini, l'effet correcteur de la fertilisation (concentration en éléments nutritifs) a ensuite été analysé en faisant porter l'effort sur la phase de récupération post-asphyxique. Le comportement du système racinaire a été étudié très précisément ; les mesures classiques de croissance pondérale et de composition minérale des organes ont été confrontés aux mesures d'échanges gazeux foliaires et d'absorption ionique racinaire.

\section{MATÉRIEL ET MÉTHODES}

Des grains de blé, variété «Fidel» (provenance Ringot), imbibés une nuit dans de l'eau sous aération constante, sont placés dans des cristallisoirs dont le fond est recouvert de papier filtre. La germination est effectuée en enceinte climatique à $15-16{ }^{\circ} \mathrm{C}$ sous un éclairement de 250 à $300 \mu \mathrm{E} . \mathrm{m}^{-2} . \mathrm{s}^{-1}, 12 \mathrm{~h}$ par j. Après une semaine $\left(7^{\mathrm{e}} \mathrm{j}\right)$, les plantes sont disposées individuellement sur des flacons contenant $500 \mathrm{ml}$ de solution nutritive ou privée d'éléments nutritifs (solution $\mathrm{CaSO}_{4} 0,5 \mathrm{mM}$ ). Cette solution est renouvelée 1 à 3 fois par jour par injection de 100 à $200 \mathrm{ml}$ de milieu neuf, la périodicité de l'apport de solution augmentant avec la croissance de la plante. Une circulation de gaz (azote ou air) assure le maintien d'une concentration très faible $\left(<0,1 \mathrm{mg} .1^{-1}\right)$ ou saturante en oxygène dans le milieu racinaire ; la séparation des racines et des parties aériennes des plantes est réalisée au moyen d'une cloison en PVC qui maintient la plante et des conduits capillaires servant à la circulation des gaz et de la solution nutritive. Les conditions de croissance en enceinte climatisée sont identiques à celles de la germination.

La solution nutritive est du type Coïc et est utilisée à la concentration normale $(1 / 1)$ ou diluée au dixième $(1 / 10)$; dans ce dernier cas, la concentration en chélate de fer est maintenue à la valeur normale. La composition est la suivante pour les macroéléments ( $\mathrm{mM})$ : $\mathrm{KNO}_{3}: 3,8 ; \quad \mathrm{KH}_{2} \mathrm{PO}_{4}: 0,8 ; \mathrm{K}_{2} \mathrm{HPO}_{4}: 0,3$; $\mathrm{Ca}\left(\mathrm{NO}_{3}\right)_{2}: 3,1 ; \mathrm{NH}_{4} \mathrm{NO}_{3}: 2 ; \mathrm{NaCl}: 0,2 ; \mathrm{MgSO}_{4}$ : 1,5 et pour les oligoéléments $\left(\mu \mathrm{g} \mathrm{l}^{-1}\right): \mathrm{Mn}: 485 ; \mathrm{Zn}$ : $210 ; \mathrm{Cu}: 60 ; \mathrm{B}: 255$; Mo : 25 ; Fe : 1800.

Pendant la $1^{\text {re }}$ semaine de culture hydroponique $\left(7^{\mathrm{e}}\right.$ $14^{\mathrm{e}} \mathrm{j}$ ), les plantes sont maintenues sur milieu aéré ; puis un lot est placé en asphyxie par circulation d'azote dans le milieu racinaire pendant $1\left(14^{\mathrm{e}}-21^{\mathrm{e}} \mathrm{j}\right)$ à 3 semaines $\left(14^{\mathrm{e}}-35^{\mathrm{e}} \mathrm{j}\right)$ selon les cas. Un lot normalement aéré sert de témoin. Les prélèvements pour les mesures de croissance sont hebdomadaires à partir du $15^{\mathrm{e}} \mathrm{j}$ et portent sur 4 plantes par lot expérimental. Le nombre de répétitions de chaque série de traitements est précisé dans les résultats.

Les masses de substance fraîche des différents organes (feuilles, talles, racines séminales et adventives) sont mesurées à chaque prélèvement. Les masses de matière sèche correspondantes sont obtenues après passage à l'étuve à $65^{\circ} \mathrm{C}$ jusqu'à masse constante. Les compositions minérales (macro- et oligo-éléments) sont déterminées sur des fractions aliquotes selon les méthodes classiques d'analyse des végétaux.

L'absorption ionique des plantes individuelles en éléments $\mathrm{N}, \mathrm{P}$ et $\mathrm{K}$ est déterminée en mesurant la consommation d'eau et d'éléments nutritifs dans le milieu racinaire pendant une semaine. Afin d'éviter les pertes d'eau par évaporation, le milieu nutritif est balayé par des gaz (air ou azote) préalablement saturés en eau à la température de la pièce de culture.

Les variations de croissance de chaque plante sont également obtenues de manière non destructive par la mesure des échanges gazeux photosynthétiques (fixation de $\mathrm{CO}_{2}$, transpiration) de l'ensemble des parties aériennes. La photosynthèse nette et la transpiration sont mesurées dans une chambre d'assimilation en plexiglas d'un volume de $10 \mathrm{l}$, alimentée en circuit ouvert. L'analyseur de $\mathrm{CO}_{2}$ à infra-rouge (ADC 225 Mark III) et l'hygromètre à point de rosée (General Eastern 1100 DP) sont reliés à un système d'acquisition de données (Hewlett-Packard HP 3054 DL) qui assure le traitement des signaux. Les mesures sont effectuées dans les conditions de température et d'éclairement des enceintes de culture, en respectant le 
port normal des feuilles, le système racinaire étant maintenu dans le flacon de culture avec circulation d'air ou d'azote. Ce système a l'avantage de permettre les mesures dans les conditions normales de croissance.

Pour chaque variable mesurée, la signification statistique des traitements est obtenue par analyse de variance et comparaisons orthogonales selon le dispositif expérimental adopté.

\section{RÉSULTATS}

\section{A. Effet de la concentration du milieu nutritif}

\section{Croissance}

La privation d'éléments nutritifs (culture sur solution $\mathrm{CaSO}_{4} 0,5 \mathrm{mM}$ ) provoque, dès l'établissement d'une asphyxie racinaire continue de 2 semaines, des effets si drastiques sur la croissance que les masses de matière (fraîche ou sèche) des lots témoins ou asphyxiés sont voisines, mais la distribution de cette matière est très différente. Le développement du système racinaire est plus faible sous asphyxie, mais se fait au profit des racines adventives qui constituent 70 p. 100 de la masse totale des racines (résultats non présentés). L'utilisation d'un milieu nutritif dilué (solution Coïc 1/10) évite d'imposer à la plante une contrainte qui occulte l'action de l'asphyxie racinaire. Dans la suite du travail, les effets de l'asphyxie sur ce milieu ont été comparés à ceux observés sur un milieu concentré (solution Coïc $1 / 1$ ).

La dilution du milieu nutritif réduit faiblement la croissance du système aérien des témoins et beaucoup plus celle des plantes asphyxiées pendant 2 semaines (tabl. 1). L'asphyxie a un effet plus marqué en solution diluée $(-45$ p. 100) qu'en solution normale ( -30 p. 100). Les effets les plus spectaculaires se manifestent sur les racines. Les témoins cultivés sur solution diluée montrent un surdéveloppement considérable des racines séminales et adventives. L'asphyxie racinaire inhibe considérablement la croissance racinaire sur solution diluée (tabl. 1). La croissance des racines des témoins montre une variabilité importante qui n'est pas observée en conditions d'asphyxie ; les racines sur solution normale peuvent représenter une proportion de la masse de matière fraîche totale qui varie de 14 p. 100 dans le cas présent jusqu'à $25-30$ p. 100 dans d'autres répétitions alors qu'en solution diluée $(1 / 10)$ la variation est de 45 à 55 p. 100 .

Quelle que soit la concentration du milieu nutritif, le critère le plus constant et caractéristique de l'asphyxie racinaire apparaît être la proportion de racines adventives dans la masse totale des racines. Elle atteint 70 à 80 p. $100 \mathrm{chez}$ les plantes asphyxiées contre 30 à 45 p. 100 chez les témoins (tabl. 1).

Comme dans les essais sur sol (GuYOT \& PRIOUL, 1985), l'asphyxie affecte peu la vitesse d'apparition des feuilles et des talles. Même à l'issue de 3 semaines d'asphyxie, la comparaison des séquences de talles montre seulement un léger retard dans l'apparition de la $5^{\text {e }}$ talle principale. Des talles secondaires $\left(T_{12}, T_{22}\right.$ et $T_{31}$ ) sont manquantes en raison d'une plus faible disponibilité de la matière sèche. Les masses des talles principales $\left(T_{1}, T_{2}\right.$ et $T_{3}$ pour l'essentiel) représentent, quel que soit le traitement, une proportion constante de la masse totale des parties aériennes quand cette masse est supérieure à $0,7 \mathrm{~g}$ frais. Les écarts de masse observés sur les talles entre plantes témoins et asphyxiées reflètent donc les écarts de masse de matière totale des parties aériennes. Les feuilles dont la croissance s'est effectuée pendant la période d'asphyxie (feuilles 4 et suivantes) atteignent leur plateau de croissance en même temps que les témoins, mais ont une masse de matière fraîche et des dimensions (longueur et largeur) significativement plus fai-

TABLEAU 1

Effet de la concentration de la solution nutritive (1/1 ou l/10) et d'une asphyxie racinaire continue sur l'accumulation et la répartition de la matière fraîche chez le blé au stade jeune. L'asphyxie est appliquée à partir du $14^{e} j$ et les plantes sont récoltées 2 semaines plus tard $3 \sigma^{e} j$ après germination). Moyennes de 4 plantes et analyse de variance des effets de l'asphyxie et de la concentration de la solution nutritive (Probabilité du test $F ; N S$ : différences non significatives au seuil de risque $5 \%$ ).

Effect of nutrient solution concentration $(1 / 1 \mathrm{vs} 1 / 10)$ and continuous root anaerobiosis on fresh matter accumulation and partitioning in voung wheat seedlings. Plants were subjected to anaerobiosis from day 14 after germination and harvested two weeks later (day 36 ). Means of four replicates and two-way analysis of variance of anaerobiosis and solution concentration effects (Probability of F-test; NS : non-significant at $5 \%$ probability level).

\begin{tabular}{|c|c|c|c|c|c|c|c|}
\hline & & \multicolumn{3}{|c|}{ Parties aériennes } & \multicolumn{3}{|c|}{ Racines } \\
\hline & & $\begin{array}{l}\text { Masse de matière } \\
\text { fraîche }(\mathrm{g})\end{array}$ & $\begin{array}{l}\text { Talles dans la } \\
\text { masse de matière } \\
\text { fraîche des } \\
\text { parties aériennes } \\
(\% 0)\end{array}$ & $\begin{array}{l}\text { Eau par unité } \\
\text { de masse de } \\
\text { matière sèche } \\
\left(\mathrm{g} \cdot \mathrm{g}^{-1}\right)\end{array}$ & $\begin{array}{l}\text { Masse de matière } \\
\text { fraîche }(\mathrm{g})\end{array}$ & $\begin{array}{c}\text { Racines dans la } \\
\text { masse de matière } \\
\text { fraîche totale } \\
(\%)\end{array}$ & $\begin{array}{l}\text { Racines adventives } \\
\text { dans la masse } \\
\text { totale de racines } \\
\text { fraîches }(\%)\end{array}$ \\
\hline \multirow[t]{2}{*}{ Témoin } & $1 / 1$ & 5,81 & 58,5 & 5,68 & 0,94 & 14,0 & 33,4 \\
\hline & $1 / 10$ & 4,85 & 56,1 & 5,98 & 6,12 & 55,2 & 32,3 \\
\hline \multirow[t]{2}{*}{ Asphyxie } & $1 / 1$ & 4,05 & 58,9 & 4,72 & 1,45 & 26,5 & 78,7 \\
\hline & $1 / 10$ & 2,67 & 50,8 & 4,11 & 1,37 & 33,9 & 76,7 \\
\hline \multirow{3}{*}{\multicolumn{2}{|c|}{$\begin{array}{l}\text { Asphyxie } \\
\text { Concentration } \\
\text { Asphyxie } \times \\
\text { Concentration }\end{array}$}} & $<0,01 \%$ & NS & $<0,01 \sigma_{0}$ & $0,01 \%$ & $0,8 \quad \%$ & $<\quad 0,01 \%$ \\
\hline & & $0,04 \%$ & $1,4 \%$ & NS & $<0,01 \%$ & $0,01 \%$ & NS \\
\hline & & NS & NS & $1,2 \%$ & $<0,01 \%$ & $0,01 \%$ & NS \\
\hline
\end{tabular}


bles ( -20 p. 100). Ces effets s'expliquent par une élongation cellulaire moindre due à la baisse de teneur en eau (tabl. 1).

\section{Composition minérale}

Dans les témoins normalement aérés, la dilution du milieu nutritif au 1/10 réduit la teneur en $N$ et $P$ des parties aériennes de près de 40 p. 100 , alors que celle des autres éléments est peu affectée $(\mathrm{K}, \mathrm{B}$ et $\mathrm{Fe})$ ou parfois augmentée ( $\mathrm{Ca}, \mathrm{Mg}, \mathrm{Mn}, \mathrm{Zn}$ ) (cf. tabl. 2). L'asphyxie diminue les teneurs de la plupart des éléments, mais cet effet et celui de la dilution du milieu nutritif ne s'additionnent pas strictement; en conditions asphyxiantes, l'écart des teneurs entre solution normale $(1 / 1)$ et diluée $(1 / 10)$ est faible, à l'exception de l'azote.

\section{TABLEAU 2}

Effet de l'asphyxie racinaire sur la composition minérale des parties aériennes de plantes cultivées sur solution nutritive normale (1/1) ou diluée (1/10). Asphyxie à partir du $14^{e} j$, récolte $36^{e} j$. Moyenne de 4 plantes.

Effect of root anaerobiosis on shoot mineral content in plants grown on normal $(1 / l)$ or diluted $(1 / 10)$ solution. Anaerobiosis from $14^{\text {th }}$ day, sampling $36^{\text {th }}$ day. Mean of 4 plants.

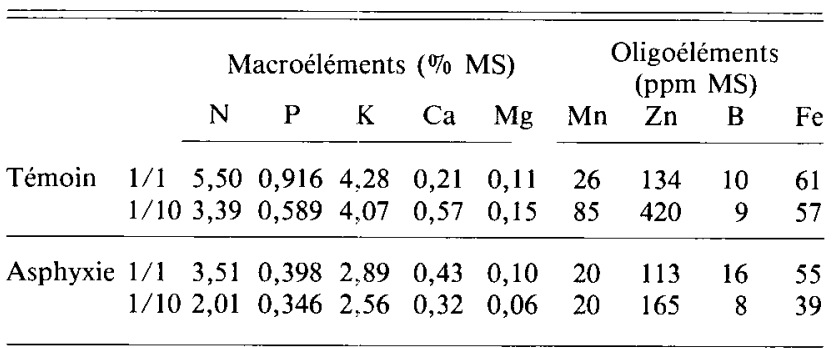

D'une manière générale, l'asphyxie provoquée par circulation d'azote gazeux dans le milieu racinaire produit des effets similaires à ceux de l'ennoyage sur sol, mais les effets observés sur solution nutritive normale sont beaucoup moins marqués. La dilution du milieu amplifie l'effet de l'asphyxie sur la croissance, celle des racines en particulier, et les teneurs en éléments des parties aériennes sont comparables à celles obtenues dans notre expérimentation sur sol (GUYOT \& PRIOUL, 1985). C'est la raison pour laquelle la suite du travail a porté sur les cultures en solution diluée.

\section{B. Effet de la durée de l'asphyxie et reprise post- asphyxique}

L'analyse précise des cinétiques de croissance a été entreprise en portant l'accent sur les critères apparus les plus importants au cours des essais précédents : développement du système racinaire et, en particulier, proportions relatives de racines séminales et de racines adventives. En outre, en vue d'une meilleure comparaison avec les essais sur sol, l'effet de la reprise de l'aération après une semaine d'asphyxie a été examiné.

\section{Croissance}

Les résultats d'une expérience représentative montrent (fig. 1) que la réduction de croissance en matière fraîche des parties aériennes due à l'asphyxie est décelable après une semaine et s'amplifie nettement dans les 2 semaines suivantes (fig. 1a). Le ralentissement initial est dû, pour l'essentiel, à la diminution brutale de la teneur en eau des feuilles des plantes asphyxiées (fig. 2a), ce qui explique les faibles différences de masse de matière sèche avec les témoins, pendant les 2 premières semaines (fig. $1 \mathrm{~b}$ ). Le rétablissement de l'aération après une semaine d'asphyxie provoque une légère reprise de la croissance en matière fraîche, mais qui est essentiellement due au relèvement de la teneur en eau ; les feuilles en croissance retrouvent alors une taille voisine de celle des témoins. A la fin de l'asphyxie, l'accumulation plus grande de matière sèche dans les parties aériennes des plantes asphyxiées par rapport à celles asphyxiées puis réaérées n'a pas été retrouvée dans les expériences ultérieures (cf. tabl. 3).
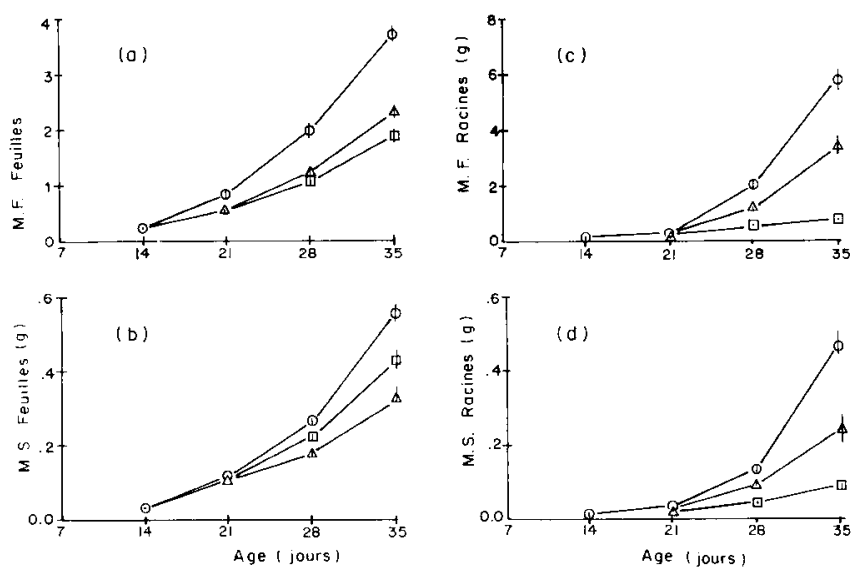

Figure 1

Effet de la durée de l'asphyxie racinaire sur l'évolution de la masse de matière fraîche ( $a$ et $c$ ) et sèche $(b$ et $d)$ des parties aériennes et des racines de blé : plantes témoins $\bigcirc$ ou soumises à une asphyxie racinaire d'une semaine $\left(14^{e}\right.$ au $\left.2 I^{e} j\right) \Delta$ ou de 3 semaines $\left(14^{e}\right.$ au $35^{e}$ j) $\square$. Chaque point représente la moyenne de 4 mesures, le trait vertical l'écart-type de la moyenne.

Effect of root anaerobiosis on variation of fresh (a and c) and dry $(b$ and $d)$ weight of shoots and roots of wheat seedlings : control $\bigcirc$; plants submitted to root anaerobiosis for one week (day 14 to 21) $\Delta$, or three weeks (day 14 to 35) $\square$. Means of four replicates; vertical bar : standard error.

C'est surtout au niveau racinaire que se manifestent les changements les plus importants (fig. $1 \mathrm{c}$ et $\mathrm{d}$ ). L'asphyxie prolongée inhibe presque complètement la croissance racinaire tandis que la levée de la contrainte induit, dès la semaine suivante, une reprise très forte. La teneur en eau des racines est moins variable que celle des parties aériennes, mais on retrouve la même tendance à une teneur en eau plus faible après une asphyxie prolongée (fig. 2b). 

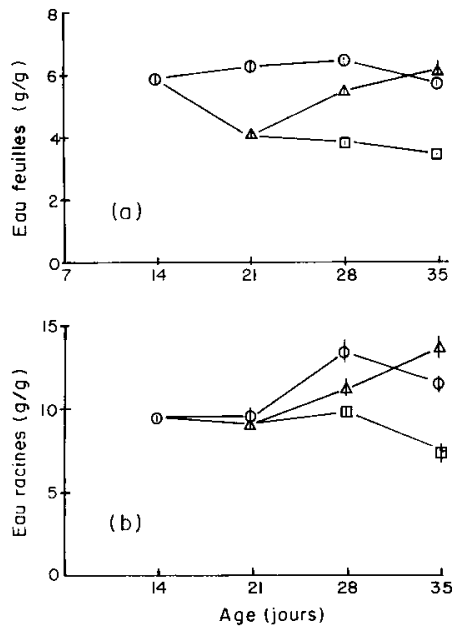

Figure 2

Teneur en eau, rapportée à la masse de matière sèche, des parties aériennes et des racines de plantes témoins 0 ou soumises à une asphyxie racinaire d'une semaine $\left(14^{e}\right.$ au $\left.21^{e} j\right) \Delta$ ou de 3 semaines (14 au $\left.35^{e} j\right) \square$. Chaque point représente la moyenne de 4 mesures, le trait vertical l'écart-type de la moyenne.

Water content (water per unit of dry matter) of shoots and roots; control $\bigcirc$; plants submitted to root anaerobiosis for one week (day 14 to 21) $\Delta$ or three weeks (day 14 to 35) L」. Each symbol represents the mean of four replicates; vertical bar : standard error.

L'asphyxie exerce un effet différentiel spectaculaire sur les 2 types de système racinaire. La croissance des racines séminales est complètement bloquée par l'asphyxie et les possibilités de reprise après seulement une semaine sont très réduites (fig. 3a); après 3 semaines d'asphyxie, la sénescence de ces racines est très marquée : couleur jaune et brune et début de dégénérescence. Par contre, quel que soit le traitement, des racines adventives de 1 à $2 \mathrm{~mm}$ de diamètre apparaissent à partir du $21^{\mathrm{e}} \mathrm{j}$ et poursuivent leur croissance dans les semaines suivantes (fig. 3b). Ces racines ont une longueur plus réduite en condition asphyxique que chez les témoins. Ce sont elles qui
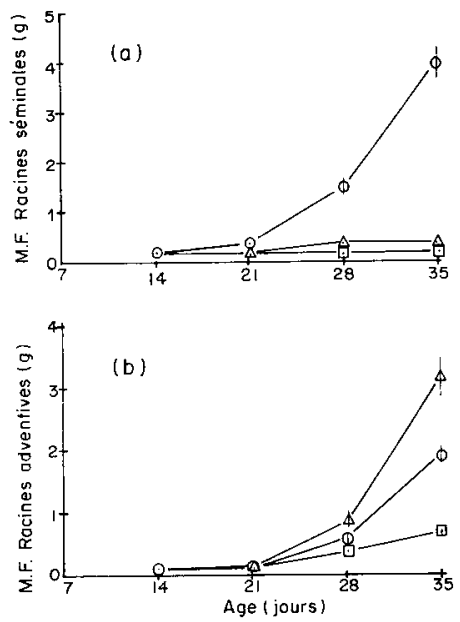

Figure 3

Effet de l'asphyxie racinaire sur l'évolution de la masse de matière fraîche des racines séminales (a) et adventives $(b)$ en fonction du temps; plantes témoins $U$, ou soumises à une asphyxie racinaire

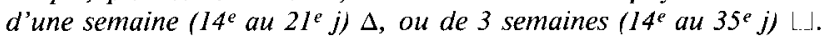
Chaque point représente la moyenne de 4 mesures, le trait vertical l'écart-type de la moyenne.

Effect of root anaerobiosis on variation in the fresh weight of seedling (a) and adventitious (b) roots; control $O$, plants submitted to root anaerobiosis for one week (day 14 to 21) $\Delta$ or three weeks (day 14 to 35$) \square$. Means of four replicates and standard error.

assurent la reprise du système racinaire lors du rétablissement de l'aération après une semaine d'asphyxie.

Ce type d'essais qui a été répété de nombreuses fois (12 essais) révèle une évolution très reproductible de la croissance (tabl. 3), avec toutefois une grande variabilité de la proportion de racines dans la masse de matière totale chez les témoins ; mais dans tous les cas, le pourcentage de racines adventives dans la masse totale de racines reste l'indice le plus sûr d'une asphyxie racinaire. Après 3 semaines de contrainte, on obtient toujours de l'ordre de 80 p. 100 de racines adventives chez les plantes asphyxiées, contre 40 p. 100 seulement chez les témoins. Une autre cons-

\section{TABLEAU 3}

Effet de la durée de l'asphyxie racinaire sur la croissance pondérale et la répartition de la matière fraîche chez le blé âgé de 33 à $36 j$. Le traitement est appliqué pendant une semaine suivie de 2 semaines d'aération, ou pendant 3 semaines. Moyenne de 12 essais et intervalle de confiance de la moyenne au seuil de risque $5 \%$.

Effect of time of root anaerobiosis on fresh matter accumulation and partitioning in 33 to 36-day old wheat seedlings. Oxygen was depleted in the root medium for one week followed by two weeks of re-aeration, or during three weeks. Means of 12 successive experiments and standard error at $5 \%$ probability level.

\begin{tabular}{|c|c|c|c|c|c|c|}
\hline & \multicolumn{3}{|c|}{ Parties aériennes } & \multicolumn{3}{|c|}{ Racines } \\
\hline & $\begin{array}{l}\text { Masse de matière } \\
\text { fraîche }(\mathrm{g})\end{array}$ & $\begin{array}{l}\text { Talles dans la } \\
\text { masse de matière } \\
\text { fraîche des } \\
\text { parties aériennes } \\
(\%)\end{array}$ & $\begin{array}{l}\text { Eau par unité } \\
\text { de masse de } \\
\text { matière sèche } \\
\left(g . g^{-1}\right)\end{array}$ & $\begin{array}{l}\text { Masse de matière } \\
\text { fraîche }(\mathrm{g})\end{array}$ & $\begin{array}{l}\text { Racines dans la } \\
\text { masse de matière } \\
\text { fraîche totale } \\
(\%)\end{array}$ & $\begin{array}{l}\text { Racines adventives } \\
\text { dans la masse } \\
\text { totale de racines } \\
\text { fraîches }(\%)\end{array}$ \\
\hline $\begin{array}{l}\text { Témoin } \\
\text { Asphyxie } \\
1 \text { semaine }+\end{array}$ & $4,33 \pm 0,68$ & $43,6 \pm 18,6$ & $6,21 \pm 0,56$ & $4,77 \pm 1,11$ & $50,4 \pm 5,0$ & $37,0 \pm 5,0$ \\
\hline $\begin{array}{l}2 \text { semaines aération } \\
3 \text { semaines }\end{array}$ & $\begin{array}{l}2,68 \pm 0,66 \\
1,88 \pm 0,29\end{array}$ & $\begin{array}{l}31,8 \\
34,5 \pm 15,6\end{array}$ & $\begin{array}{l}6,83 \pm 1,57 \\
4,40 \pm 0,53\end{array}$ & $\begin{array}{l}2,50 \pm 0,98 \\
0,84 \pm 0,16\end{array}$ & $\begin{array}{l}45,4 \pm 7,8 \\
30,7 \pm 1,6\end{array}$ & $\begin{array}{l}78,4 \pm 7,0 \\
77,1 \pm 4,5\end{array}$ \\
\hline
\end{tabular}


tatation à caractère général est que la reprise de croissance après asphyxie concerne essentiellement le système racinaire, les parties aériennes des plantes asphyxiées une semaine ne commençant à se distinguer des lots placés en asphyxie prolongée qu'après 2 semaines d'aération.

\section{Photosynthèse}

La photosynthèse nette totale, mesurée en plaçant l'ensemble des parties aériennes dans la chambre d'assimilation, en veillant à respecter le port normal des feuilles, sous un éclairement et à une température identiques à ceux de la culture, permet de mesurer les gains nets instantanés de carbone pendant la phase diurne (fig. 4a).
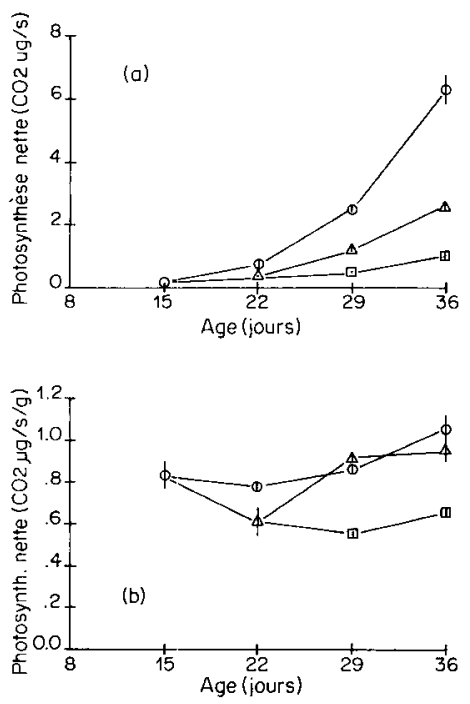

Figure 4

Photosynthèse nette par plante (absorption nette de $\mathrm{CO}_{2}$ ) (a) et photosynthèse nette spécifique ( $\mathrm{CO}_{2}$ par unité de masse de tissu frais) (b) de plantes témoins $\cup$, ou soumises à une asphyxie racinaire d'une semaine $\left(15^{e}\right.$ au $\left.22^{e} j\right) \Delta$, ou de 3 semaines (15e au $36^{e}$ jour) $L \perp$. Chaque point représente la moyenne de 4 mesures, le trait vertical l'écart-type de la moyenne.

Net photosynthesis per plant (a) and specific net photosynthesis $\left(\mathrm{CO}_{2}\right.$ per unit of fresh tissue weight) (b) : in control plants $\mathrm{O}$, or plants submitted to root aanerobiosis for one week (day 15 to 22) $\Delta$, or three weeks (day 15 to 36) $\square$. Means of four replicates and standard error.

L'asphyxie empêche presque complètement l'augmentation de photosynthèse par la plante. Le rétablissement de l'aération après une semaine de contrainte permet une reprise à vitesse toutefois plus faible que chez les témoins. Les photosynthèses nettes spécifiques (par unité de masse de matière fraîche de tissu aérien) (fig. 4b) qui ont les dimensions d'un taux de croissance, sont sensiblement constantes chez les témoins, ce qui est cohérent avec l'existence d'une croissance quasi exponentielle. L'asphyxie réduit notablement les capacités photosynthétiques, donc le taux de croissance. La suppression de la contrainte conduit au retour à la valeur du témoin alors qu'en asphyxie prolongée, la valeur se maintient à un niveau faible.
Les pertes d'eau par transpiration suivent l'évolution des photosynthèses nettes, mais la transpiration spécifique (par unité de masse de matière fraîche de tissu aérien) des plantes après asphyxie reste inférieure à celle du témoin (résultats non présentés).

\section{Composition minérale}

Dans les parties aériennes, les teneurs de tous les éléments minéraux totaux, à l'exception du calcium, varient de manière similaire: l'asphyxie racinaire induit une baisse brutale des teneurs (divisées par un facteur 2 à 4) qui demeurent constantes ou diminuent faiblement après la $1^{\text {re }}$ semaine d'asphyxie chez les plantes placées en asphyxie continue. Le rétablissement de l'aération permet une récupération de la richesse minérale à la valeur du témoin en 2 semaines (fig. 5a à e).

L'asphyxie racinaire entraîne une disparition presque totale de l'azote nitrique et du phosphate des parties aériennes et, contrairement aux éléments totaux correspondants, le rétablissement de l'aération ne ramène pas les teneurs aux valeurs du témoin (fig. $6 \mathrm{a}$ et b).

Dans les racines, les teneurs en éléments totaux évoluent d'une manière comparable à celle des parties aériennes, avec cependant une récupération moins rapide pendant la $1^{\text {re }}$ semaine de rétablissement de l'aération mais qui est complète au bout de 2 semaines (fig. 5f à j). Chez les plantes asphyxiées, les teneurs des racines adventives tendent à être plus élevées que celles des racines séminales, même sous asphyxie prolongée.

Ce sont les racines séminales des plantes asphyxiées qui accusent la plus forte diminution de teneur en $\mathrm{N}$ nitrique, laquelle ne parvient pas à se rétablir avec la reprise de l'aération (fig. 6c). Dans les racines adventives, on assiste à une élévation progressive de la concentration en nitrates, même sous asphyxie continue. A la $5^{\text {e }}$ semaine de croissance, la récupération sous asphyxie continue correspond aux $2 / 3$ du témoin et est totale dans les racines ayant subi une seule semaine d'asphyxie. Les variations de concentration en phosphate sont moins amples que celles des nitrates, mais la seule récupération observable concerne les racines adventives des plantes soumises à une asphyxie d'une semaine (fig. 6d).

Ces résultats confirment la carence en macroéléments totaux des parties aériennes et des racines consécutives à l'asphyxie. Le marqueur le plus intéressant est la teneur en $N$ nitrique dont la réponse à l'asphyxie est amplifiée par rapport à celle de l'azote total et révèle un comportement différentiel des racines séminales et adventives; le relèvement des teneurs dans ces dernières sous asphyxie suggère un comportement adaptatif. Les teneurs en nitrates et, à un moindre degré, en phosphate expriment nettement la réponse physiologique post-asphyxique de la plante qui accorde la priorité au système racinaire au détriment des parties aériennes, résultat en bonne concordance avec les mesures de croissance.

L'analyse des consommations ioniques (tabl. 4) explique les variations de composition minérale. Au cours de la $1^{\text {re }}$ semaine d'asphyxie, l'absorption d'ions est au moins réduite de moitié à cause, d'une part, du 

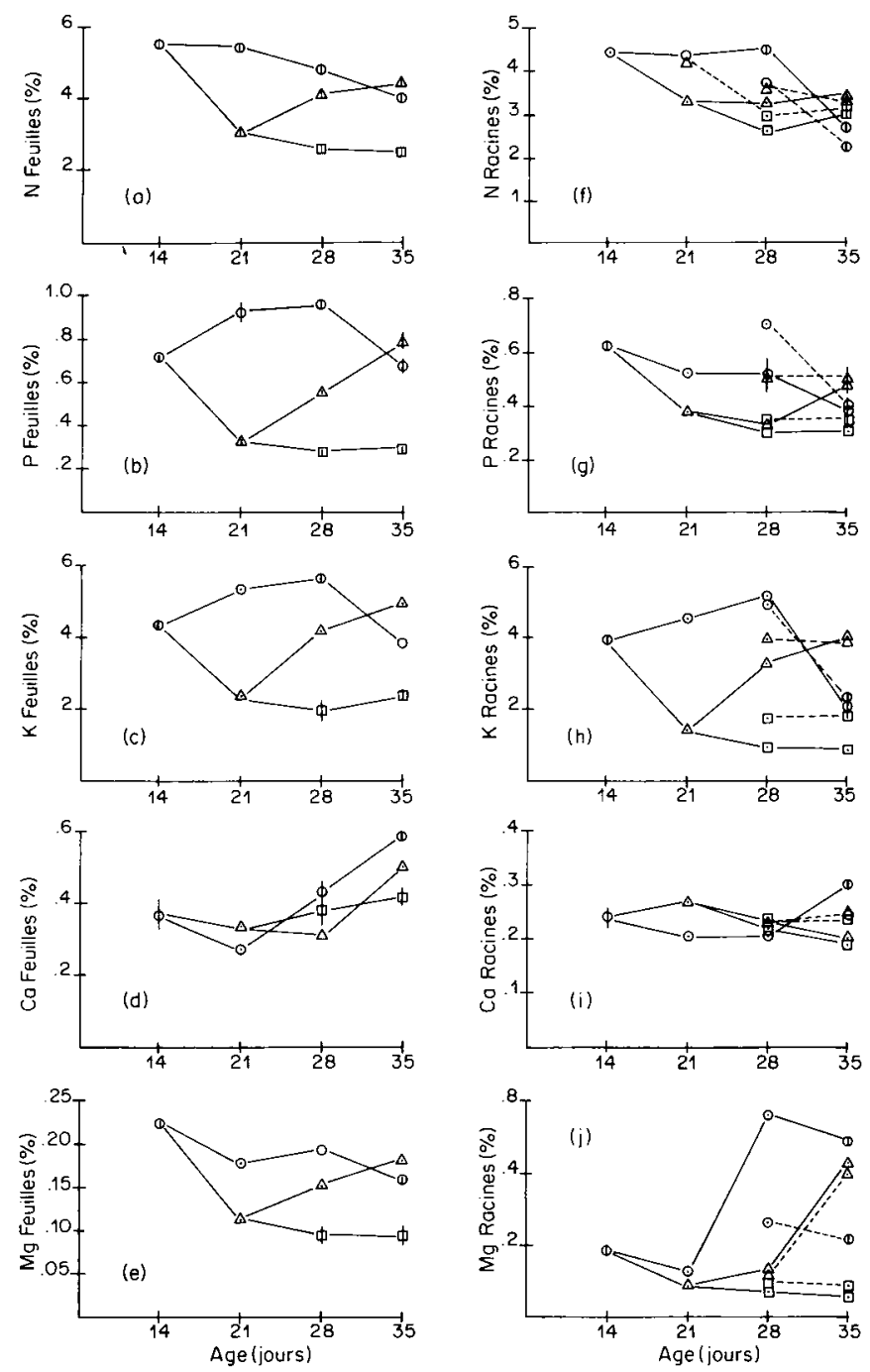

Figure 5

Teneur en éléments minéraux $(N, P, K, C a, M g)$, rapportée à la masse de matière sèche, des parties aériennes ( $a$ à e) et des racines (f à j) séminales (_ ou adventives (_- - ) de plantes cultivées sur solution nutritive diluée (1/10). Plantes témoins $\bigcirc$ ou soumises à une asphyxie racinaire d'une semaine (14 au $21^{e}$ j) $\Delta$ ou de 3 semaines $\left(14^{e}\right.$ au $35^{e}$ jour $) \square$. Chaque point représente la moyenne de 5 essais, le trait vertical l'écart-type de la moyenne.

Mineral content $(N, P, K, C a, M g$ ), on a dry matter basis, of wheat seedling shoots ( $a$ to e) and seedling (-) or adventitious (-_roots (f to j). Plants grown in dilute nutrient solution (1/10). Control $O$; plants submitted to root anaerobiosis for one week (day 14 to 21) $\Delta$ or three weeks (day 14 to 35) $\square$. Means of five experiments and standard error.

flux transpiratoire plus faible et, d'autre part, de la diminution de concentration en nutriments par unité de masse d'eau transpirée. Ce dernier point suggère l'inhibition partielle des processus d'absorption des ions. La reprise de l'aération conduit à un rétablissement rapide de fonctions qui restent un peu inférieures à la normale.

\section{DISCUSSION}

L'asphyxie racinaire permet de reproduire tous les effets de l'ennoyage, ce qui confirme que le défaut d'approvisionnement en oxygène est bien l'agent cau-
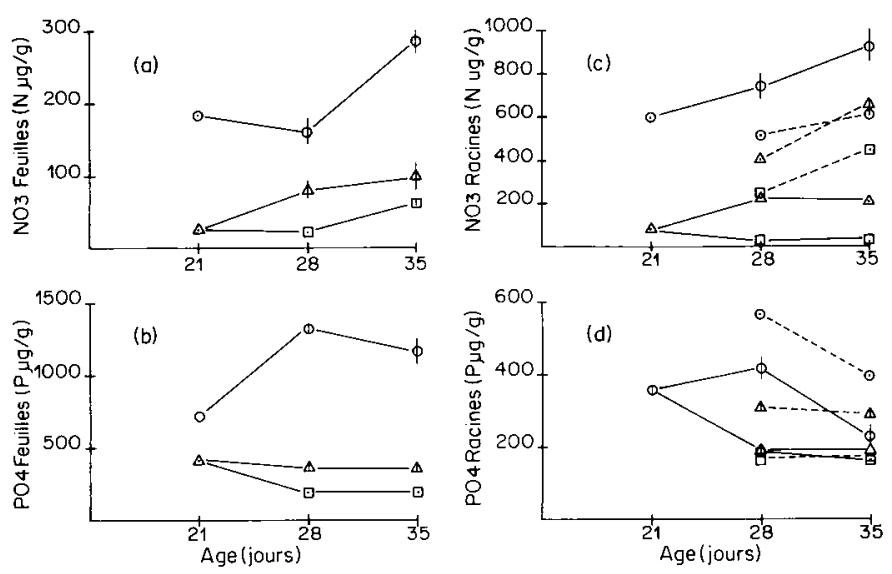

Figure 6

Teneur en azote nitrique $\mathrm{N}\left(\mathrm{NO}_{3}^{-}\right)$et en phosphate $\mathrm{P}\left(\mathrm{PO}_{4}^{--}\right)$, rapportée à la masse de matière fraîche, des parties aériennes $(a, b)$ et des racines $(c, d)$ séminales (__ ou adventives (_-_-) de plantes cultivées sur solution nutritive diluée $(1 / 10)$. Plantes témoins $)$ ou soumises à une asphyxie racinaire d'une semaine $\left(14^{e}\right.$ au $\left.21^{e} \mathrm{j}\right) \Delta$ ou de 3 semaines $\left(14^{e}\right.$ au $\left.35^{e} j\right)$ $\square$. Chaque point représente la moyenne de 5 essais, le trait vertical l'écart-type de la moyenne.

Nitric nitrogen $\mathrm{N}\left(\mathrm{NO}_{3}^{-}\right)$and inorganic phosphorus $\left.\mathrm{P}_{\left(\mathrm{PO}_{4}^{-}\right.}^{-}\right)$content (on a fresh matter basis), of shoots ( $a, b)$ and seedling (__ or adventitious (-__-) roots $(c, d)$. Plants grown in dilute nutrient solution (1/10). Control $\bigcirc$; plants submitted to root anaerobiosis for one week (day 14 to 21) $\Delta$ or three weeks (day 14 to 35) $\square$. Means of five experiments and standard error.

sal des phénomènes observés (TROUGHT \& DREW, 1981 ; DREW, 1983). On observe toutefois des différences dans l'intensité du phénomène : une semaine d'ennoyage sur sol étant équivalente à 3 semaines d'asphyxie sur solution nutritive normale. D'autre part, la croissance après reprise de l'aération reste plus perturbée sur sol, ce qui s'explique probablement par la difficulté de rétablissement d'un drainage normal en raison de la modification de structure du sol induite par le traitement.

Les effets macroscopiques sur la croissance peuvent être interprétés par la séquence d'événements suivante : l'asphyxie racinaire entraine primairement une réduction du métabolisme respiratoire (SAGLIO et al., 1980) qui affecte tous les mécanismes consommateurs d'énergie, dont l'absorption ionique active, l'entrée d'eau associée et l'exportation dans le xylème vers les parties aériennes (LOUGHMAN, 1978; GRIGNON \& TOURAINE, 1981 ; TOURAINE \& GRIGNON, 1981). La réduction de la teneur en eau empêche l'ouverture complète des stomates et entraîne progressivement la baisse de la photosynthèse nette (fig. 4). Dans un premier temps ( $1^{\text {re }}$ semaine), la croissance en matière sèche continue au même rythme, mais le déséquilibre entre les besoins et les entrées d'ions (tabl. 4) conduit à une carence dans les parties aériennes. Cette carence, en particulier celle en nitrates, provoque une réduction de la conductivité hydraulique et de la turgescence qui induit la baisse du taux de croissance (RADIN \& BOYER, 1982).

Dans ce contexte, on peut expliquer le rôle spécifique d'un apport d'éléments nutritifs, puisqu'en renforçant les flux passifs, l'effet de la carence se trouve limité.

Cette interprétation ne rend cependant pas totalement compte du comportement du système racinaire 
TABLEAU 4

Absorption ionique et transpiration hebdomadaires de plantes témoins ou soumises à une asphyxie racinaire, cultivées sur solution nutritive diluée $(1 / 10)$. Effet du rétablissement de l'aération (2e semaine) après une semaine d'asphyxie. Moyenne de 4 plantes. Les valeurs en italiques représentent les quantités d'éléments par $m \mathrm{~m}$ d'eau transpirée.

Ion absorption per week of wheat seedlings grown in dilute nutrient solution (1/10). Effect of oxygen depletion (1st week) and re-aeration (2nd week) of the root medium. Means of four replicates.

\begin{tabular}{|c|c|c|c|c|c|c|c|}
\hline & $\mathrm{NH}_{4}^{+}(\mathrm{mg} \mathrm{N})$ & $\mathrm{NO}_{\overline{3}}^{-}(\mathrm{mg} \mathrm{N})$ & $\mathrm{P}\left(\mathrm{mg} \mathrm{P}_{2} \mathrm{O}_{5}\right)$ & $\mathrm{K}(\mathrm{mg})$ & $\mathrm{Ca}$ (mg) & $\mathrm{Mg}(\mathrm{mg})$ & $\begin{array}{c}\text { Eau transpirée } \\
(\mathrm{ml})\end{array}$ \\
\hline \multicolumn{8}{|c|}{$\begin{array}{l}\text { Consommation ionique } \\
\text { (mg/semaine) ou } \mu g \cdot \mathrm{ml}^{-l} \\
\text { transpirée } \\
\quad 1^{\text {re }} \text { semaine }\end{array}$} \\
\hline Témoin & 94 & $85^{1,67}$ & $\begin{array}{l}1,49 \\
74\end{array}$ & $\begin{array}{ll}4,52 \\
230\end{array}$ & $\begin{array}{l}0,11 \\
17\end{array}$ & $\begin{array}{c}0,18 \\
9\end{array}$ & 20,7 \\
\hline Asphyxie & $\begin{array}{l}0,89 \\
60\end{array}$ & $\begin{array}{l}0,87 \\
58\end{array}$ & 24 & ${ }_{I I 3}^{1,80}$ & $\begin{array}{l}0,14 \\
4\end{array}$ & $\begin{array}{l}0,07 \\
5\end{array}$ & 16,8 \\
\hline \multicolumn{8}{|l|}{$2^{\mathrm{e}}$ semaine } \\
\hline Témoin & $\begin{array}{l}2,09 \\
82\end{array}$ & $\begin{array}{l}3,84 \\
153\end{array}$ & $\begin{array}{l}1,99 \\
79\end{array}$ & 299 & $\begin{array}{l}0,28 \\
12\end{array}$ & $\begin{array}{l}0,23 \\
9\end{array}$ & 25,7 \\
\hline $\begin{array}{l}\text { Asphyxie et reprise } \\
\text { aération }\end{array}$ & 2,06 & $121^{2,81}$ & $93^{1,48}$ & $\begin{array}{l}5,73 \\
248\end{array}$ & $\begin{array}{l}0,25 \\
8\end{array}$ & $\begin{array}{l}0,19 \\
8\end{array}$ & 24,1 \\
\hline
\end{tabular}

et de la séquence des événements après reprise de l'aération. En effet, le présent travail, aussi bien sur sol (GUYOT \& PRIOUL, 1985) que sur solution nutritive, montre que l'asphyxie présente un arrière-effet très important sur l'équilibre racines-parties aériennes qui aboutit à une surcompensation de la croissance racinaire au profit des seules racines adventives. Par ailleurs, l'examen de l'évolution du système racinaire pendant la période d'asphyxie permet de soulever plusieurs questions : comment expliquer la croissance importante des racines adventives et le maintien en survie des racines séminales alors que la concentration en oxygène dans le milieu est très basse $\left(<0,1 \mathrm{mg} .1^{-1}\right)$ ? Comment la concentration en nutriments, nitrates en particulier, peut-elle avoir un effet protecteur aussi puissant ? La compréhension de ces phénomènes doit être envisagée dans le cadre des réactions d'adaptation du système racinaire à l'asphyxie : examen de l'importance du transport interne d'oxygène à partir des parties aériennes via les aérenchymes des racines adventives (LUXMOORE \& STOLZY, 1969) et évaluation des adaptations métaboliques (McMANMON \& CRAWFORD, 1971 ; JACKSON et al., 1982). Cet aspect est spécifiquement traité dans un autre travail qui complète celui-ci (PrIOUL \& GUYOT, 1985).
En conclusion, les conséquences de l'asphyxie sont fortement dépendantes de la concentration en éléments nutritifs : on peut les effacer presque complètement avec les milieux nutritifs optimaux définis pour les cultures hydroponiques (type Coïc $1 / 1$ ). Ce point confirme bien l'effet d'un apport d'engrais, sous forme soluble, observé sur sol et démontre que la compensation peut être très efficace à condition de tendre, comme dans le cas des solutions nutritives, vers des concentrations en nutriments en équilibre avec celles des flux transpiratoires, afin de solliciter au minimum les processus actifs d'absorption de la racine. Des essais complémentaires au champ sur la relation dose d'engrais apporté paraissent nécessaires pour évaluer l'efficacité de la correction en fonction de l'intensité de la contrainte (TroughT \& DREW, 1981).

Reçu le 15 juin 1984. Accepté le 12 avril 1985.

\section{REMERCIEMENTS}

Mme R. Zelwer, M. J. Y. Gautier, M. G. Vuillemin et Mme M. Coupeau (Rhône-Poulenc Recherches, Centre de Recherches de la Croix de Berny) sont remerciés pour leur assistance technique efficace.

\section{RÉFÉRENCES BIBLIOGRAPHIQUES}

Armstrong W., 1979. Aeration in higher plants. Adv. Bot. Res., 7, 225-332.

Drew M. C., 1983. Plant injury and adaptation to oxygen deficiency in the root environment : review. Plant Soil, 75, 179-199.

Grignon C., Touraine B., 1981. Les fonctions de transport de la racine. I. Les fonctions de prélèvement. Physiol. Vég., 19, 443-462.

Guyot C., Prioul J. L., 1985. Correction par la fertilisation minérale des effets de l'ennoyage sur le blé d'hiver. I. Expérimentation sur sol. Agronomie, 5 (8), 743-750.
Jackson M. B., Herman B., Goodenough A., 1982. An examination of the importance of ethanol in causing injury to flooded plants. Plant Cell Environ., 5, 163-172.

Loughman B. C., 1978. Metabolic factors and the utilization of phosphorus by plants, 155-174. In R. Porter \& D. W. Fitzsimons : Phosphorus in the environment : its chemistry and biochemistry. Elsevier, Amsterdam.

Luxmoore R. J., Stolzy L. H., 1969. Root porosity and growth responses of rice and maize to oxygen supply. Agron. J., 61, 202-204. 
McManmon M., Crawford R. M. M., 1971. A metabolic theory of flooding tolerance : the significance of enzyme distribution and behaviour. New Phytol., 70, 299-306.

Morisset C., Raymond P., Mocquot B., Pradet A., 1982. Adaptation des végétaux à l'hypoxie et l'anoxie. Bull. Soc. Bot. Fr., 129, 73-89.

Prioul J.-L., Guyot C., 1985. Role of oxygen transport and nitrate metabolism in the adaptation of wheat plants to root anaerobiosis. Physiol. Vég., 23 (2), sous presse.

Radin J. W., Boyer J. S., 1982. Control of leaf expansion by nitrogen nutrition in sunflower plants. Role of hydraulic conductivity and turgor. Plant Physiol., 69, 771-775.
Saglio P. H., Raymond P., Pradet A., 1980. Metabolic activity and energy charge of excised maize root tips under anoxia. Plant Physiol., 66, 1053-1057.

Touraine B., Grignon C., 1981. Les fonctions de transport de la racine. II. Les fonctions d'exportation. Physiol. Vég., 19, 581-610.

Trought M. C. T., Drew M. C., 1980. The development of waterlogging damage in young wheat plants in anaerobic solution cultures. J. Exp. Bot., 31, 1573-1585.

Trought M. C. T., Drew M. C., 1981. Alleviation of injury to young wheat plants in anaerobic solution cultures in relation to the supply of nitrate and other inorganic nutrients. J. Exp. Bot., 32, 509-522. 